\title{
PROCESO MIGRATORIO DE LOS PERUANOS: \\ ¿QUÉ PASA CON LOS PLANES DE ACOGIDA PARA LOS RETORNADOS?
}

\author{
Norma Velásquez Rodríguez ${ }^{*}$ \\ Universidad Católica Sedes Sapientiae \\ nvelasquez@ucss.edu.pe
}

ReSUMEN: La presente investigación describe los programas de retorno y acogida (asumidos por el Gobierno Peruano) y determina las herramientas para mejorar el diseño de políticas de retorno de los connacionales peruanos. Es muy importante que el gobierno peruano trabaje en mejorar las herramientas de participación y la estructura de los programas de retorno

* Norma Velásquez Rodríguez es doctora en Economía con el reconocimiento de Palmas Universitarias. Tiene una maestría en Gerencia de Proyectos de Ingeniería y un Máster Business Administration (MBA). Se ha especializado en análisis del mercado laboral con énfasis en las políticas públicas para la generación del empleo, oferta laboral y gestión de políticas migratorias. Es investigadora y analista de proyectos sociales y de inversión con alto impacto a nivel nacional e internacional. Es consultora para la Corporación Financiera Internacional-Banco Mundial (IFC), el Programa Conjunto de las Naciones Unidas, la Organización Internacional para las Migraciones y colaboradora externa de la Organización Internacional del Trabajo (OIT). Asimismo, ha participado como investigadora principal en proyectos financiados por la Unión Europea. Además, ha participado en el Stage en Investigación Social realizado en la Universitá degli Studi di Trento en Italia. Actualmente, participa en el Observatorio Andino de Migración, Interculturalidad y Codesarrollo, y en el diseño de políticas laborales migratorias para la Región Andina. A su vez, se desempeña como docente asociada de la Universidad Católica Sedes Sapientiae. 
y acogida, debido a que, actualmente, no se dan los efectos esperados del plan y la coordinación entre los países participantes.

El documento analiza un marco teórico, los programas de retorno de la Unión Europea, los programas de acogida y se concluye con un balance. La metodología empleada ha sido uso de información secundaria y entrevistas a los principales actores que intervienen en el proceso de migración.

Palabras Clave: retorno, políticas y programas de retorno, migración de peruanos, proceso migratorio.

\section{PERUVIAN PEOPLE IMMIGRATION PROCESS:}

\section{WHAT HAPPENS WITH THE ASSISTANCE PLANS FOR PEOPLE WHO RETURN THEIR COUNTRY?}

\footnotetext{
ABSTRACT: This research describes the return program (on the side of the Peruvian Government) and determines the tools for improving return policies and processes of Peruvian people. It is necessary that the Peruvian government starts improving the involvement tools and the structure of the return program. At the moment, the expected effects of the plan and the coordination between the participating countries are not evidenced.

The paper discusses a theoretical framework on migration, return programs of the European Union and host programs and it concludes with an assessment. The methodology uses secondary information and interviews with the main people involved in the migration process.

KeYwords: return, Return policies and programs, peruvian migrations, the migration process.
} 
PROCESO MIGRATORIO DE LOS PERUANOS:

\section{INTRODUCCIÓN}

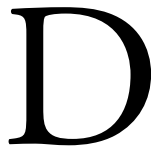

entro del proceso migratorio un tema clave de analizar es el retorno.

En el documento publicado por el Banco Mundial «Datos sobre migración y remesas $2011 »$, se indica que en todo el mundo hay más de 215 millones de migrantes internacionales. ${ }^{1}$ Estos migrantes han remitido a los países en desarrollo cerca de US\$325 000 millones de dólares durante el año 2010, lo que excede ampliamente el volumen de los flujos de ayuda oficial y constituye más del $10 \%$ del Producto Interno Bruto (PIB) de muchos países en desarrollo. ${ }^{2}$ Asimismo, muchos de ellos tienen planes de retornar a sus países de origen. Sin embargo, ¿qué implicancias tiene el retorno tanto para el país de acogida como para el país de origen?, ¿existen políticas en el Perú que ayuden en el proceso de retorno?

En el presente artículo nos enfocaremos en las políticas de acogida que tiene nuestro país, rescatando la importancia de desplegar una mejor gestión en el proceso migratorio, sobre todo, en el ámbito laboral. De manera que permita un diseño de políticas migratorias como también la generación de programas que ordenen las migraciones. Esto permitirá lograr un desarrollo y transformación en los mercados de trabajo, así como formativos, de modo que sean coherentes con las necesidades del país. El documento contempla generar una visión general de los programas y estrategias realizados en Europa, sobre la base del Fondo Europeo para el Retorno, denominado «Programa Solidaridad y Gestión de Flujos Migratorios». Dicho programa está siendo aplicado por los países europeos. En este caso particular, se ha

1 Para el caso de los peruanos emigrados sin retorno; desde 1990 suman más de 2 millones.

2 Los datos sobre migración y remesas 2011 fueron recopilados por Dilip Ratha, Sanket Mohapatra y Ani Silwal del Grupo del Banco Mundial. Unidad de Migración y Remesas del Banco Mundial. 
revisado la aplicación realizada en España con el financiamiento del Fondo Europeo para el Retorno en el periodo 2008-2013. También se discuten las estrategias de promoción del programa de retorno; así como las barreras que pudieran impedir su aplicación.

\section{MARCO METODOLÓGICO}

El estudio ha sido realizado mediante el uso de fuentes bibliográficas. Se han analizado procesos y desarrollo de programas efectuados en el marco de la crisis internacional. Entre los problemas encontrados en el proceso de elaboración del estudio destacan los siguientes: (a) la carencia de unificación de la terminología de retorno; (b) los países carecen de información detallada sobre datos demográficos, cualificaciones de los inmigrantes, entre otros. La información que se ha podido recabar sobre los programas de retorno no siembre incluye la nacionalidad y lugar de retorno; (c) sobre los beneficiarios de los programas de retorno voluntario, la información es escasa, atomizada y muy general; $y(d)$ en los países andinos se ha encontrado poca información sobre el seguimiento de los beneficiarios de subvenciones de retorno.

\section{MARCO TEÓRICO}

El tema de migración de retorno, es un área relativamente nueva de la migración. No tiene un significado estándar en la política o el derecho nacional o internacional. Se han propuesto diferentes tipologías de retorno para describir el nivel de desarrollo de los países vinculados por la migración y el retorno, el tiempo pasado en el país de origen, la intención de los inmigrantes ante el resultado efectivo o el entorno sociológico del retornado.

La revisión de la literatura permite indicar que es escasa la información sobre los conceptos y la gestión de la migración de retorno. 
Si se desea abarcar países específicos, como en el caso de los países andinos, las fuentes se vuelven más difíciles de encontrar. Muchas veces la definición se torna ambigua, en la medida en que dicho fenómeno es considerado como el punto final en el proceso migratorio de una persona. Para muchos autores como Ballesteros (2006) estudiar la migración de retorno resulta complicada, porque hay elementos que son importantes de considerar como, por ejemplo, el que no siempre la persona considera al retorno como un punto final en su proceso migratorio, pues puede existir otra migración; o el país considerado de origen como punto final de la migración de retorno, puede ser un país de tránsito para otra migración, siendo difícil la manera de medirlo. Al hablar de migración de retorno también deben incluirse las diversas causas del regreso, los diferentes tipos de retorno que pueden contribuir a discriminar entre situaciones, los retornos en modalidades desiguales, las formas de integración del retornado en la comunidad y, sobretodo, en su hogar, los cambios sociales que origina el retorno. Muchos autores como Gmelch (1980), Gaillard ${ }^{3}$ (1994) indican que si bien es cierto existen diferentes causas para retornar para cada emigrante, también existen factores básicos que influyen en esta decisión. Muchas veces esta decisión no depende de factores económicos, sino radica en la nostalgia por su país de origen, la forma de vida, los lazos familiares y amigos.

En la investigación realizada por Franco (2010: 8) se indica que en los estudios empíricos, como los mencionados en el trabajo de Berninghaus y Siefert-Vogt (1989), la mayoría de los migrantes planifican solo una estadía

3 Gaillard sistematizó las diferentes razones que influyen en la decisión de retornar: (a) aspectos relacionados a la familia; (b) retiro de la vida laboral; (c) acumulación del capital para establecer algún negocio en el país de origen; (d) posibilidad de trabajar en el país de origen; (e) problemas o inconformidad en el país de destino; (f) motivos culturales (g) motivos personales. 
temporal en el país de destino, aproximadamente no menor a tres años ni mayor a seis. Sin embargo, en muchos casos, se aplaza la fecha del regreso y la migración de retorno se hace menos probable después de algún tiempo. Cerase (1974) desarrolló una tipología con base en criterios socioculturales, tomando como ejemplo a los inmigrantes italianos retornados de Estados Unidos. (Ver Tabla 1)

\begin{tabular}{|c|c|}
\hline $\begin{array}{l}\text { Tipología de } \\
\text { retorno }\end{array}$ & Descripción \\
\hline $\begin{array}{l}\text { Retorno del } \\
\text { fracaso }\end{array}$ & $\begin{array}{l}\text { Las características que lo distinguen son: la emigración } \\
\text { se dio en un breve tiempo, no hubo una adaptación en la } \\
\text { sociedad de destino, el trabajo que se desarrolló se dio bajo } \\
\text { condiciones precarias y con un sentido de malestar, ante esta } \\
\text { perspectiva el emigrante decide regresar a su lugar de origen. }\end{array}$ \\
\hline $\begin{array}{l}\text { Retorno de } \\
\text { conservadurismo }\end{array}$ & $\begin{array}{l}\text { Identifica a emigrantes retornados que se fueron con el } \\
\text { objetivo de trabajar y ahorrar. Es por ello, que el regreso se } \\
\text { da cuando el migrante obtiene los insumos necesarios para } \\
\text { mejorar su calidad de vida en su lugar de origen. }\end{array}$ \\
\hline $\begin{array}{l}\text { Retorno de } \\
\text { retiro }\end{array}$ & $\begin{array}{l}\text { Es el que se produce cuando se alcanza la jubilación, cuando } \\
\text { se tiene una edad avanzada o después de alcanzar los objetivos } \\
\text { propios de la edad adulta. Aquí el migrante añora su lugar de } \\
\text { origen y su hogar. Por lo tanto, toma la decisión de regresar. }\end{array}$ \\
\hline $\begin{array}{l}\text { Retorno de } \\
\text { innovación }\end{array}$ & $\begin{array}{l}\text { En este tipo de migración de retorno se identifica a los } \\
\text { emigrantes que aspiran con el regreso, para elevar su nivel } \\
\text { de vida, pero tomando como referencia las pautas de la } \\
\text { sociedad donde han sido migrantes, experiencias vividas } \\
\text { y aprendizajes, convirtiéndose en actores de cambio en su } \\
\text { sociedad de origen. }\end{array}$ \\
\hline
\end{tabular}

\section{Tabla 1. Tipos de migración de retorno identificados por Cerase}

Fuente: Franco José. Los que regresan: migración de retorno en la región de los Altos de Jalisco. Seminario: Movilidad Espacial de la Población y Migración. FLASCO. Sede México. Tesis para optar el grado de Maestro en Población y Desarrollo. Agosto 2010, p. 19. 
Uno de los investigadores que más ha contribuido a los estudios sobre la migración de retorno es Durand (2004). En un estudio sobre jóvenes trató de esclarecer la tipología del migrante de retorno y comprender el fenómeno social que viven algunos jóvenes. Durand indica que el retorno del migrante de un país considerado como desarrollado puede ser algo contradictorio e incluso incomprensible.

El retorno se encuentra delimitado por procesos que involucran directamente al sujeto y a los vínculos sociales que le rodean. La migración de retorno pone en cuestión el proceso final a la migración. La decisión de retornar puede estar determinada por las vivencias y experiencias del migrante durante su estancia, además de los cambios económicos, políticos, sociales y culturales que vivió en la zona de destino y en la de origen. La tipología de la migración de retorno que plantea Durand (2004) contempla las causas que motivan el regreso de los sujetos a su lugar de origen, como se muestra en la presente tabla:

\begin{tabular}{|l|l|}
\hline Tipología & Características \\
\hline $\begin{array}{l}\text { Migración } \\
\text { voluntaria }\end{array}$ & $\begin{array}{l}\text { Hay algunos factores que contribuyen a este tipo de } \\
\text { migración, tales como el ahorro de dinero por el migrante. } \\
\text { Al cabo de una estancia de algunas décadas, estos deciden } \\
\text { regresar a su lugar de origen. Los sujetos sociales que regresan } \\
\text { son en su mayoría personas jubiladas yensionadas; le siguen } \\
\text { otros de edad intermedia, cuando aumentan en el país de } \\
\text { origen las oportunidades de mejorar la calidad de vida. }\end{array}$ \\
\hline $\begin{array}{l}\text { Trabajadores } \\
\text { temporales }\end{array}$ & $\begin{array}{l}\text { Los migrantes se encuentran condicionados por programas } \\
\text { específicos o, bien, por un contrato, el cual determina su } \\
\text { retorno, como, por ejemplo, en México el Programa Bracero, } \\
\text { entre México y Estados Unidos (1942-1964) y los Programas } \\
\text { de visas H2a y H2b de trabajadores temporales para la } \\
\text { agricultura y los servicios entre otros. }\end{array}$ \\
\hline
\end{tabular}




\begin{tabular}{|l|l|}
\hline Tipología & Características \\
\hline $\begin{array}{l}\text { Retorno transge- } \\
\text { neracional }\end{array}$ & $\begin{array}{l}\text { Se trata del retorno, ya no del migrante, sino de su } \\
\text { descendencia: hijos, nietos, bisnietos, un fenómeno cada vez } \\
\text { más relevante y que empieza a ser estudiado. }\end{array}$ \\
\hline Retorno forzado & $\begin{array}{l}\text { La historia está llena de casos de retorno forzado de pueblos } \\
\text { enteros por razones políticas y raciales. }\end{array}$ \\
\hline $\begin{array}{l}\text { Retorno } \\
\text { voluntario }\end{array}$ & $\begin{array}{l}\text { Término que se atribuye al migrante que, por diversas } \\
\text { circunstancias, no pudo establecer vínculos sociales, por } \\
\text { lo que su capital cultural y social ha disminuido. Y es que } \\
\text { las experiencias son determinantes para decidir el retorno. } \\
\text { Al no acceder a un empleo que se imaginó seguro -en un } \\
\text { país donde supuestamente la mano de obra lo encuentra } \\
\text { fácilmente- y al toparse en cambio con la violación a los } \\
\text { derechos humanos: el racismo, la incapacidad para adaptarse } \\
\text { en un país que no es el suyo, el migrante decide retornar a } \\
\text { su país. }\end{array}$ \\
\hline
\end{tabular}

Tabla 2. Tipologías de migración de retorno atribuidas a Durand (2004) Fuente: Durand, Jorge. Ensayo teórico sobre la migración de retorno. Cuadernos Geográficos, 2004.

De otro lado la Organización Internacional para las Migraciones (OIM, 2008) propone clasificar a la migración de retorno en tres categorías. Considero importante señalarlas en la presente tabla:

\begin{tabular}{|l|l|}
\hline Tipología & Características \\
\hline $\begin{array}{l}\text { Voluntarios sin } \\
\text { obligación }\end{array}$ & $\begin{array}{l}\text { Cuando los inmigrantes deciden en cualquier momento } \\
\text { volver a su país de origen por voluntad propia y por su } \\
\text { cuenta. }\end{array}$ \\
\hline
\end{tabular}


PROCESO MIGRATORIO DE LOS PERUANOS:

\begin{tabular}{|l|l|}
\hline Tipología & Características \\
\hline $\begin{array}{l}\text { Voluntarios } \\
\text { obligados }\end{array}$ & $\begin{array}{l}\text { Cuando se encuentran al final de su periodo de } \\
\text { protección temporal y no pueden permanecer en el país, } \\
\text { por lo cual eligen volver por su propia voluntad. }\end{array}$ \\
\hline Involuntarios & $\begin{array}{l}\text { Son los que deben salir de un país en cumplimiento de } \\
\text { una orden de deportación dictada por las autoridades } \\
\text { del Estado de destino. }\end{array}$ \\
\hline
\end{tabular}

Tabla 3. Tipología de la migración de retorno según OIM (2008)

Existen teorías sociológicas que explican la migración de retorno. Entre las que considero importante destacar, por su relevancia en el tema, son las siguientes: la teoría fenomenológica desarrollada por Schutz, la teoría del conflicto social, el enfoque estructural y el enfoque transnacional.

De otro lado, la teoría económica ayuda mucho a poder explicar las principales razones que llevan a las personas a desarrollar el ciclo migratorio. La «teoría neoclásica» es la que ha analizado, en mayor medida, las razones de la migración. Entre los investigadores más renombrados, dentro de la perspectiva neoclásica, destacan Todaro (1969); Borjas (1989); Piore, M. (1979); Durand (1996); Espinosa (1998); y Sassen (1988). Bajo el enfoque neoclásico el migrante retornado puede verse como una persona para la cual el proceso migratorio no tuvo los beneficios esperados. Existió un error de cálculo en donde no se aprovecharon los beneficios de la migración y no se recompensó el capital humano de la manera esperada.

En cuanto a la «nueva economía de la migración» considera la situación de migrar no solo como una decisión individual aislada, sino que esta se hace en unidades más amplias de grupos humanos, en donde se 
evalúan los riesgos y beneficios de migrar (Stark y Taylor 1988). En la nueva economía de la migración se introduce el concepto de "privación relativa». ${ }^{4}$ La migración de retorno para la nueva economía de la migración es vista como el resultado lógico de una estrategia calculada, donde los migrantes cumplen las metas y objetivos fijados por la familia y regresan al hogar como consecuencia de una experiencia exitosa en el extranjero. Las remesas son parte de la estrategia para mejorar el nivel de ingresos.

\section{CONCEPTOS $^{5}$}

Según las «definiciones académicas», retorno es la acción o efecto de retornar; se refiere al regreso o devolución al punto de partida. En este sentido, y, de acuerdo con la División de Estadísticas de Naciones Unidas, migrantes retornados son las personas que retornan a su país de origen después de haber sido inmigrantes ${ }^{6}$ en otro país y que tratan de quedarse en su país por un año al menos.

4 Dicho concepto explica que si bien la migración es una estrategia en la que el grupo familiar envía trabajadores al extranjero tomando en cuenta las remesas futuras, no solo lo hace para mejorar sus ingresos en términos absolutos sino también para mejorarlos relativamente respecto a otros hogares de la misma comunidad sin importancia del monto real de los mismos y en consecuencia, reducir su desventaja relativa.

5 En este apartado se van a considerar conceptos para retorno partiendo de una definición estrictamente lingüística. Se han seleccionado diversas definiciones centradas en materia migratoria, manejadas por los principales organismos internacionales especializados; así como por los programas de retorno gubernamentales que se han analizado en este trabajo.

6 Sea por un periodo corto o largo. Esta definición excluye de la consideración de retornados a las personas que se vuelven a su país de origen para estar un breve periodo de tiempo (de visita familiar, vacaciones, etc.). 
Por otro lado, el Parlamento Europeo y el Consejo de la Unión Europea, mediante la Directiva de Retorno ${ }^{7}$ (2008/115/EC), proponen una definición de retorno. Esta es la que sigue:

Proceso de vuelta de un nacional de un tercer país, bien sea en acatamiento voluntario de una obligación de retorno, bien de modo forzoso a su país de origen, o un país de tránsito con arreglo a acuerdos de readmisión comunitarios o bilaterales o de otro tipo, u otro tercer país al que el nacional de un tercer país decida volver voluntariamente y en el cual será admitido. (Diario Oficial de la Unión Europea del 24/12/2008 p. L348/101)

Entre otras categorías del retorno destacan tres. Estas son las siguientes: (a) retorno voluntario: según la OIM, se trata del regreso, independiente o asistido, al país de origen, de tránsito u a un tercer país, basado en la libre voluntad de la persona que regresa; (b) retorno voluntario asistido: tras el análisis de los programas de retorno implementados por diversos países de acogida, así como de algunos de los principales países de emisión de flujos migratorios, el retorno asistido es el que se produce mediante algún tipo de ayuda aportada por el estado, una ONG o un Organismo Internacional; (c) retorno productivo: sobre la base de definiciones extraídas de los programas de retorno productivo, implementados por organismos internacionales $y$ estados, ${ }^{8}$ así como de documentos de Naciones Unidas y de la Comisión Europea sobre Migración y Desarrollo, se trata de las siguientes: retorno al

7 Dada el 16 de diciembre de 2008 por el Parlamento Europeo y El Consejo de la Unión Europea relativa a normas y procedimientos comunes en los Estados miembros para el retorno de los nacionales de terceros países en situación irregular

8 Se ha analizado fundamentalmente programas de España, Francia, Alemania y Bélgica. 
país de origen con la idea de abrir o expandir un negocio; retorno al país de origen para emprender un proyecto empresarial, ya sea microempresa o empresa familiar, proyecto productivo comunitario o cooperativa ligada al codesarrollo, o bien una mediana empresa.

\section{LOS PROGRAMAS DE RETORNO DE LA UNIÓN EUROPEA}

A continuación presentaremos los principales programas de retorno estructurados en la Unión Europea en los últimos años. Se hace referencia a los programas realizados en España y el proceso de implementación de proyectos y actividades en relación al retorno.

Las últimas estadísticas difundidas por el Banco Mundial (2011: 38) indican que la inmigración al 2010 a Europa y Asia Central es de 27,3 millones o $6,8 \%$ de la población (comparado con los 215,8 millones o 3,2\% a nivel mundial). De ellos el $56,7 \%$ son mujeres inmigrantes, un $0,8 \%$ son refugiados. La Unión Europea, ha generado una serie de programas o $y$ estrategias referidos al retorno, siendo su política ${ }^{9}$ la generación de un espacio de "Libertad, Seguridad y Justicia», donde se busca favorecer y garantizar la libre circulación de las personas en el territorio de la Unión Europea (ciudadanos de la Unión Europea y de terceros Estados). Por ello, ha desarrollado varios mecanismos de integración, en el año 1992 se establecen nuevas formas de cooperación en materia de asilo y migración a

9 El Art. 2 del TUE (antiguo art. A del Tratado de Maastricht). La política de la Unión Europea busca «Mantener y desarrollar la Unión como un espacio de Libertad, seguridad y justicia, en el que esté garantizada la libre circulación de las personas conjuntamente con medidas adecuadas respecto al control de las fronteras exteriores, el asilo, la inmigración y la prevención y la lucha contra la delincuencia» 
través del Tratado de Masstricht, ${ }^{10}$ donde se incluyó una política generando el Fondo Social Europeo. Siete años después, el Tratado de Ámsterdam (Ocaña 2012) estableció la base legal para una política de migración común en un área de libertad, seguridad y justicia, ${ }^{11}$ incluyendo la libre circulación de las personas, el control de las fronteras exteriores, asilo, inmigración y cooperación judicial en materia civil. Para 1999, en el Consejo de Tampere ${ }^{12}$ se definieron las políticas para la creación de un área de libertad, seguridad y justicia estableciendo una política común en materia de migración y asilo siendo un argumento crucial para la cooperación con los países de origen, un régimen común de asilo, el trato justo para nacionales de terceros países y la gestión de los flujos migratorios. Para el año 2001, en el comunicado de la Comisión Europea sobre Política Común de Inmigración Ilegal, ${ }^{13}$ se señala que la Política de Retorno es una parte integral y crucial de la lucha contra la inmigración ilegal. El documento tuvo como objetivo reunir en un marco coherente los elementos clave de una política común en materia

10 Véase para mayor información el portal Tratado de la Unión Europea. Disponible en <http://eur-lex.europa.eu/es/treaties/dat/11992M/htm/11992M.html>. Consulta hecha en $30 / 01 / 12$.

11 El Tratado afirma que la Unión Europea se basa en los principios de libertad, democracia, respeto de los derechos humanos y de las libertades fundamentales y del Estado de Derecho. Estos principios son comunes a todos los estados miembros. En este sentido, posteriormente en 1998, el Consejo Europeo reunido en Colonia, acordó que la UE redacte y apruebe una Carta de Derechos Fundamentales.

12 Véase para mayor información el portal Parlamento Europeo, sitio web encargado de facilitar al ciudadano información concerniente a la organización, los poderes y las actividades del Parlamento Europeo. Disponible en <http:/www.europarl.europa.eu/ summits/tam_es.html>. Consulta hecha en 01/02/2012.

13 Véase para mayor información el portal Europa. Síntesis de la legislación de la UE, sitio web encargado de proveer los principales aspectos de la legislación europea. Disponible en <http://europa.eu/legislation_summaries/other/l33191_es.html>. Consulta hecha en $01 / 02 / 2012$. 


\section{NORMA VELÁSQUEZ RODRÍGUEZ}

de inmigración; exponer las futuras medidas y formas de cooperación con el fin de establecer una política estructurada de prevención y lucha contra la inmigración irregular. El documento señala que la «Política de Retorno» se debe basarse en tres elementos: (a) principios comunes, (b) normas comunes y (c) acciones comunes. Estos puntos se trataron a mayor detalle en lo que se conoce como el «Libro Verde» (2002) que busca una política común en materia de retorno. El documento resalta la necesidad de aproximación y de mayor cooperación en materia de retorno. Posteriormente, el 14 de octubre del 2002, la comunicación de la Comisión relativa a una política comunitaria de retorno tuvo en cuenta los resultados de este proceso público de audiencia, donde se bosquejó un programa concreto de nuevas medidas, buscando una acción comunitaria en materia de repatriación, por ello el 28 de noviembre del mismo año se pidió una mayor cooperación operativa entre los Estados miembros, una cooperación con terceros países más intensa estableciendo de normas comunes con el objetivo de facilitar el retorno operativo. Se aborda también el proceso de acción en relación a la gestión de retorno en los Estados miembros de la Unión Europea de nacionales de terceros países, incluyendo las etapas intermedias del retorno como la preparación y seguimiento. En el año 2004, el Programa de la Haya solicitó de manera expresa el establecimiento de normas comunes para las personas repatriadas, respetando los derechos humanos y su dignidad. La Unión Europea a través del Programa marco de Solidaridad y gestión de los flujos migratorios para el periodo $2007-2013,{ }^{14}$ buscó mejorar la gestión de

14 Comunicación de la Comisión al Consejo y al Parlamento Europeo por la que se establece un Programa marco de Solidaridad y gestión de los flujos migratorios para el periodo 2007-2013. Véase para mayor información el portal Europa. Síntesis de la legislación de la UE, sitio web encargado de proveer los principales aspectos de la legislación europea. Disponible en <http://europa.eu/legislation_summaries/justice_freedom_security/ free_movement_of_persons_asylum_immigration/114509_es.htm >. Consulta hecha en 02/02/2011. 
los flujos migratorios de la Unión Europea y reforzar la solidaridad entre los Estados miembros. ${ }^{15}$ El Programa tiene cuatro dimensiones: (a) la gestión integrada de las fronteras exteriores, con la creación de un Fondo para las Fronteras Exteriores; (b) la política de asilo, con la prolongación del Fondo Europeo para los Refugiados; (c) la integración social, cívica y cultural de los nacionales de terceros países, con la creación de un Fondo Europeo para la Integración; (d) la lucha contra la inmigración ilegal y el retorno de los nacionales de terceros países que residen ilegalmente en el territorio de la Unión Europea, con la creación de un Fondo Europeo para el Retorno. El Programa Marco Solidaridad y gestión de los flujos migratorios es de 5, 866 millones de euros para el periodo 2007-2013. Además de la dotación global, se asignarán: 285,1 millones de euros a la Agencia Frontex; ${ }^{16} 900$ millones de euros para realizar sistemas de información a gran escala y 62,3 millones de euros para la creación de un Observatorio Europeo de la Migración. La Unión Europea maneja una serie de fondos para el desarrollo de programas y proyectos para mejorar la gestión de los flujos migratorios. A continuación los detallamos a través de la siguiente tabla:

15 Se inscribe en el marco de las perspectivas financieras 2007-2013. Tiene por objeto garantizar una programación plurianual de la UE en distintos ámbitos (gestión integrada de las fronteras, política de asilo, integración y retorno).Este Programa se inscribe en la línea de los programas actuales (el Fondo Europeo para Refugiados-FER-, por ejemplo), de programas anteriores (Argo y Odysseus), y de las acciones preparatorias (como las INTI).

16 Agencia europea para la gestión de la cooperación operativa en las fronteras exteriores. Su finalidad es mejorar la gestión integrada de las fronteras exteriores de los Estados miembros de la Unión Europea 


\begin{tabular}{|l|l|}
\hline Fondo & Características \\
Fondo Europeo para los & $\begin{array}{l}\text { El programa prevé ajustar los objetivos de tal manera } \\
\text { que el FER tenga una duración hasta 2013, teniendo } \\
\text { nuevas disposiciones que entraron en vigencia el 01 } \\
\text { de enero de 2008. Esto permitirá la aplicación de la } \\
\text { puesta en marcha del III Programa Plurianual de la } \\
\text { Unión Europea para 2011-2013. }\end{array}$ \\
\hline $\begin{array}{l}\text { Fondo para las Fronteras } \\
\text { Exteriores }\end{array}$ & $\begin{array}{l}\text { Considera las recomendaciones del Programa de } \\
\text { La Haya que se centra en la necesidad de distribuir } \\
\text { las competencias entre los Estados miembros, } \\
\text { de manera equitativa, en lo relativo al control y } \\
\text { vigilancia de las fronteras exteriores. }\end{array}$ \\
\hline $\begin{array}{l}\text { Fondo Europeo para la } \\
\text { Integración }\end{array}$ & $\begin{array}{l}\text { Este fondo se llevó a cabo después de una serie de } \\
\text { proyectos pilotos sobre la integración realizados } \\
\text { desde el año 2002. }\end{array}$ \\
\hline $\begin{array}{l}\text { Fondo Europeo para el } \\
\text { Retorno }{ }^{17}\end{array}$ & $\begin{array}{l}\text { Responde a la demanda difundida en el Programa } \\
\text { de La Haya. Este fondo es materia de estudio para } \\
\text { conocer los programas y planes desarrollados en la } \\
\text { Unión Europea a favor del retorno }\end{array}$ \\
\hline
\end{tabular}

Tabla 4. Fondos de la Unión Europea para desarrollo de programas y proyectos en relación a flujos migratorios

Existen otras directivas dadas en torno al retorno. A continuación se presentarán las principales disposiciones junto a estas directivas: 


\begin{tabular}{|l|l|}
\hline Instrumentos & Comentarios \\
\hline Decisión N. & $\begin{array}{l}\text { De } 5 \text { de marzo de 2008. Establece las normas de aplicación } \\
\text { de la Decisión N. 575/2007/CE del Parlamento Europeo } \\
\text { y del Consejo por la que se establece el Fondo Europeo } \\
\text { para el Retorno para el periodo 2008-2013 como parte } \\
\text { del Programa general Solidaridad y gestión de los flujos } \\
\text { migratorios, en lo que respecta a los sistemas de gestión } \\
\text { y control de los Estados miembros, las normas sobre la } \\
\text { gestión administrativa y financiera y la elegibilidad de los } \\
\text { gastos de los proyectos cofinanciados por el Fondo (Diario } \\
\text { Oficial L 167 de 27/06/2008). }\end{array}$ \\
\hline Decisión N. & $\begin{array}{l}\text { Del 30 de noviembre de 2007, Se aplica la Decisión N. } \\
\text { 575/2007/CE del Parlamento Europeo y del Consejo, } \\
\text { en lo que respecta a la adopción de las orientaciones } \\
\text { estratégicas para el periodo 2008-2013 (Diario Oficial L } \\
\text { 330 de 15/12/2007). La presente Decisión establece las } \\
\text { orientaciones estratégicas para el periodo 2008-2013, por } \\
\text { las que se definen las prioridades de la Unión Europea y las } \\
\text { prioridades específicas de los países de la Unión Europea } \\
\text { para actuar con el fin de recibir una mayor contribución } \\
\text { financiera del Fondo para el Retorno. Dicha Decisión } \\
\text { establece cuatro prioridades: (a) apoyo al desarrollo de un } \\
\text { enfoque estratégico de la gestión del retorno; (b) apoyo } \\
\text { a la cooperación entre los países de la Unión Europea en } \\
\text { materia de gestión del retorno; (c) apoyo a herramientas } \\
\text { innovadoras específicas nacionales e internacionales para } \\
\text { la gestión del retorno y (d) apoyo a las normas europeas y } \\
\text { buenas prácticas en materia de gestión del retorno. }\end{array}$ \\
\hline
\end{tabular}




\begin{tabular}{|l|l|}
\hline Instrumentos & Comentarios \\
\hline La Directiva de $^{1}$ & $\begin{array}{l}\text { La Directiva de retorno }{ }^{17} \text { es una norma comunitaria } \\
\text { cuyo objetivo establecer procedimientos y normas } \\
\text { comunes que han de aplicarse a los Estados miembros de } \\
\text { la Unión Europea para el retorno de aquellos nacionales } \\
\text { de terceros países que se encuentren irregularmente } \\
\text { en su territorio, con ciertas excepciones. En el caso } \\
\text { de aquellos nacionales de terceros países que queden } \\
\text { excluidos del ámbito de aplicación de esta directiva, los } \\
\text { Estados miembros garantizarán que el trato y el nivel de } \\
\text { protección no sean menos favorables que los establecidos } \\
\text { en determinadas disposiciones sobre medidas coercitivas, } \\
\text { expulsión, asistencia sanitaria e internamiento. Los } \\
\text { Estados miembros respetarán siempre el principio de no } \\
\text { devolución y tendrán en cuenta el principio del interés } \\
\text { superior del niño, la vida familiar y estado de salud de la } \\
\text { persona implicada. La directiva entró en vigencia el 13 de } \\
\text { enero de 2009. }\end{array}$ \\
\hline
\end{tabular}

Tabla 5. Disposiciones y directivas entorno al retorno

\section{Nota técnica.}

1. La Directiva de retorno se basó en un acuerdo entre el Parlamento Europeo y el Consejo de la Unión Europea, tomando como antecedentes lo indicado en el Tratado de Maastrich y el de Amsterdam. La directiva es de aplicación directa. En junio 2008,el texto de compromiso de la Directiva de retorno, fue aprobado por el Consejo a nivel de representantes permanentes de los Estados Miembros (COREPER), y por Ministros de Justicia y Asuntos del Interior, y aprobado por el pleno del parlamento. Lo dispuesto en el artículo 20 establece el 24 de diciembre de 2010 como fecha límite para que los Estados miembros de la EU adecuen su legislación interna a esta norma supranacional. La Directiva otorga un plazo mayor—24 de diciembre de 2011— para

17 Directiva 2008/115/CE del Parlamento Europeo y del Consejo, de 16 de diciembre de 2008, relativa a normas y procedimientos comunes en los Estados miembros para el retorno de los nacionales de terceros países en situación irregular. Véase para mayor información el portal Europa. Sintesis de la legislación de la UE, sitio web encargado de proveer los principales aspectos de la legislación europea. Disponible en <http://europa. eu/legislation_summaries/justice_freedom_security/free_movement_of_persons_ asylum_immigration/j10014_es.html>. Consulta hecha en 01/02/2011. 
dar cumplimiento a las obligaciones de los Estados de la Unión Europea en materia de asistencia jurídica y/o representación legal gratuita a los nacionales de terceros países que interpongan acciones judiciales contra las decisiones relativas a su retorno o soliciten su revisión.

Entre los programas antes mencionados recalcamos el «Fondo Europeo para el Retorno» que busca apoyar los esfuerzos de los Estados miembros en la mejora de la gestión de todas las dimensiones del retorno. Para el periodo 2008-2013 se cuenta con una dotación total de 676 millones de euros, el Fondo forma parte del Programa General Solidaridad y Gestión de los Flujos Migratorios. El fondo financia cuatro tipos de proyectos. Estos son los siguientes: (a) desarrollo de un enfoque estratégico del retorno; (b) cooperación entre los estados miembros en materia de gestión de retorno; (c) creación de herramientas innovadoras específicas nacionales e internacionales para la gestión del retorno y (d) apoyo a las normas comunitarias y buenas prácticas para la gestión del retorno. También existen dos programas que podrán ser objeto de Subvención con fondos cofinanciados por el Fondo Europeo para el Retorno. Estos son los siguientes: (I) el Programa de Retorno Voluntario de Atención Social y (II) el Programa de retorno voluntario productivo.

\section{PROGRAMAS DE ACOGIDA EN EL PERÚ}

En este apartado se recogen propuestas realizadas sobre los procesos de atención al retorno concebido con un enfoque participativo y proactivo basado en el desarrollo de dos proyectos importantes MIGRANDINA y Programa Conjunto. Entre los mecanismos de atención al retornado identificados en el Perú destacan:

- Ministerio del Interior: ahora cuenta con una Superintendencia

Nacional de Migraciones, antes denominada Dirección General 
de Migraciones y Naturalización (DIGEMIN). Esta es la primera instancia con la cual el retornado entra en contacto, en el momento del registro de su entrada en el país. Se cuenta con una base de datos gestionada por la Unidad de Informática y Estadística que recoge información básica sobre la persona que retorna, la misma que es analizada conjuntamente con el INEI y la IOM. La información que se obtiene no ayuda mucho para el diseño de políticas sobre todo en atención al retornado

-Ministerio de Relaciones Exteriores ${ }^{18}$ : a través de la Dirección General de Comunidades Peruanas en el Exterior y Servicios Consulares gestiona acciones en materia migratoria. Entre los instrumentos desarrollados por el Ministerio en cuanto al retorno destacan los siguientes: La Encuesta a las Comunidades del Exterior (2012); «La Guía para el inmigrante peruano reciente»; el documento sobre la "Política de reforma consular, de protección, asistencia y promoción de las comunidades peruanas en el exterior»; y la nueva Ley de Inventivos Migratorios Ley N. 30001, publicada el 14.03.2013, que tiene por objeto "brindar facilidades para todos aquellos compatriotas que deseen retornar de manera definitiva para dedicarse a actividades profesionales o empresariales(...)”. Es importante recalcar el papel que realizan las oficinas consulares peruanas en el exterior que ayudan a los connacionales que voluntariamente decidan volver a residir en el Perú. Se cuenta con el Programa de Asistencia Humanitaria aprobada por Resolución Ministerial N. 1075/RE-2005, el Ministerio de Relaciones Exteriores contempla la repatriación de ciudadanos peruanos en

18 Véase para mayor información <http://www.rree.gob.pe/portal/mre.nsf>. 
situación de indigencia, enfermos y/o difunto: (para estos casos, en el caso de que los familiares se encuentren en el Perú, deberán comunicar sus demandas o presentar la respectiva solicitud a la Dirección de Asistencia al Nacional).

- Ministerio de la Producción: se ha creado el Programa CRECEMIYPE está orientado a la informacióny asesoramiento y capacitación para nuevas ideas microempresariales. No se trata de un servicio establecido exclusivamente para personas que retornan, pero sí se contempla la posibilidad de la participación de esas en el programa: el portal web que difunde este programa tiene un link directo con la Plataforma de Retorno Productivo y, por otra parte, el portal web de Servicio al Migrante del MTPE ${ }^{19}$ proporciona una pequeña información sobre esta iniciativa y un link directo con el programa.

- Ministerio de la Vivienda: cuenta con el Fondo MIVIVIENDA, que tien un programa dirigido a las personas peruanas que residen en el extranjeron para adquirir una vivienda en el Perú a través del nuevo crédito MIVIVIENDA. Así podrán adquirir una vivienda de manera directa y sin intermediarios, a través de corresponsales de alguna Institución Financiera Intermediaria (IFI) que opere en el Perú, y con los beneficios de los programas del Fondo MIVIVIENDA. La vivienda a comprar deberá al menos ser habitada por un familiar, de cualquiera de los cónyuges, hasta un segundo grado de consanguinidad.

${ }^{19}$ Antes se denominó Perú Infomigra. 
- Ministerio de Trabajo y Promoción del Empleo (MTPE): a través de un trabajo con organismos de cooperación internacional el MTPE ${ }^{20}$ ha diseñado políticas públicas en cuanto a la gestión migratoria, desarrollando primero reestructuraciones al interno del Ministerio como es el caso de una mejora del ROF y el MOF. A partir de estos cambios se creó la Dirección de Migración Laboral (DML), el órgano dependiente de la Dirección General de Promoción del Empleo, dentro de la estructura orgánica del MTPE, que asume la competencia exclusiva y excluyente en materia de migración laboral. Dentro de las funciones asignadas en el artículo $67 \mathrm{del}$ DS N. 004-2010-TR, considera acciones de retorno: (a) diseñar, proponer e implementar sistemas de información sobre potenciales trabajadores peruanos en el extranjero, migrantes, retornantes y trabajadores inmigrantes; (b) diseñar, proponer y promover mecanismos de acompañamiento y seguimiento del retornado en su proceso de reinserción laboral o autoempleo, en coordinación con la Dirección General del Servicio Nacional de Empleo.

Se creó en julio de 2010 el Servicio Perú Infomigra (hoy, Orientación al Migrante), con el objetivo de brindar información y orientación, sobre el proceso de la migración laboral. Su finalidad e facilitar una toma de decisión consciente e informada de todo lo que este proceso implica y, así, promover la empleabilidad y los derechos socio laborales. Cuenta, entre sus cuatro líneas de acción, con una específicamente dedicada a personas peruanas que desean retornar al Perú. Posee una plataforma virtual interactiva que soluciona consultas a través de una dirección de correo electrónico o a través

${ }^{20}$ Migrandina y Programa Conjunto. 
de una atención personalizada. El Ministerio cuenta también con la Dirección General del Servicio Nacional del Empleo (SENEP) que es el responsable de proponer, ejecutar, dirigir y evaluar la política y la normativa en materia de intermediación laboral pública y privada. La misma que regula, coordina, ejecuta y supervisa la articulación de los servicios en materia de promoción del Empleo y capacitación laboral que se prestan a nivel nacional, regional y local, integrados en una Red Nacional, bajo su coordinación y asistencia técnica. Es el SENEP el que se encarga de gestionar la Plataforma de Retorno Productivo ${ }^{21}$ que fue creada en el año 2010. Hasta la fecha la plataforma es usada para el diseño de perfiles de personas a retornar. El MTPE cuenta con otros programas que se han diseñado no solo para el tema migratorio, sino también para buscar una accesibilidad de empleo para más peruanos, entre estos programas destacan: el Programa Revalora Perú y el programa REMPYME.

Aparte del trabajo propio de cada Ministerio, el Proyecto MIGRANDINA de la OIT conjuntamente con el Ministerio de Relaciones Exteriores diseñaron un mecanismo que ayuda a articular la política migratoria nacional a través de la conformación de la

21 Esta plataforma fue concebida como un servicio del MTYPE con el objetivo de guiar y facilitar el retorno al mercado laboral peruano de manera productiva de aquellas personas que desean regresar al Perú tras haber emigrado al extranjero. La plataforma consiste en una base de datos a través de la cual se recoge información de las personas peruanas que se encuentran en el extranjero y que tienen intención o interés en el retorno al país. Una vez que las personas interesadas ingresan sus datos en el formulario virtual establecido al efecto, la idea es que este usuario pueda ser derivado a los programas y servicios de Promoción del Empleo y Capacitación Laboral del MTPE que se hayan estimado más adecuados a sus conocimientos, capacidades, experiencias laborales y necesidades. 
Mesa Intersectorial para la Gestión Migratoria, la misma que está legalmente institucionalizada.

- Mesa Intersectorial para la Gestión Migratoria: coordinada por el Ministerio de Relaciones Exteriores y constituido por diversas instituciones y entidades, es un instrumento creado en 2010, orientado a la coordinación interministerial e interinstitucional en materia migratoria. Fue concebida como un espacio cuyo objeto es redefinir los lineamientos y principios que deben orientar la política migratoria peruana, con un enfoque de derechos y desde una perspectiva integral. Entre sus cuatro ejes temáticos, el tercero de ellos está dedicado a peruanos que retornan del exterior. Entre los lineamientos que la Mesa ha establecido para el desarrollo de este eje se encuentran los siguientes: (a) establecer un sistema de cobertura de salud para el migrante y sus derechohabientes; (b) crear mecanismos para el acceso al sistema de previsión social de los migrantes; (c) propiciar el acceso a sistemas educativos de nuestros migrantes y sus familias; y el reconocimiento de sus estudios, títulos y certificaciones de capacitación; (d) promover el acceso a la vivienda propia de nuestros migrantes y sus familiares; (e) promover la protección, inserción y empleabilidad del migrante en el mercado laboral; (f) implementar mecanismos de facilitación para la reunificación familiar, regularización migratoria y la recuperación de la nacionalidad; y $(\mathrm{g})$ la creación de programas especiales para la reinserción de migrantes en situación de alta vulnerabilidad (tráfico ilícito de migrantes y trata de personas). 


\section{ANÁLISIS DEL POTENCIAL PERUANO RETORNADO 22}

Según el INEI (2013) en el mes de abril 2013, se registraron 200810 movimientos migratorios de entradas de peruanos al país, cifra superior en $4,9 \%$ respecto al similar mes del año 2012. En cuanto a los datos generales del retorno, según el Censo 2007 ha retornado al país 43468 retornados que residían en 2002 en el exterior. Los datos que publica el INEI indican que para el periodo 2007-2009 han retornado: 18 852, 22559 y 35090 respectivamente, sobre los cuales proporciona la siguiente información relacionada al tiempo de permanencia en el exterior:

Del total de peruanos que retornaron en el año 2007, la mayoría salió entre los años 2004-2006 (64,7\%). En el caso del retorno de peruanos en el 2008, la mayoría salió entre el periodo 20052007 (63,9\%). A su vez, los peruanos retornantes en el año 2009 salieron principalmente entre el 2006 y el año 2008 con un 68,5\% del total de peruanos retornantes en ese año y que estuvieron viviendo en el exterior de un año a más tiempo (Organización Internacional para las Migraciones et al. 2010: 41)

En cuanto a las cifras que maneja la OCDE sobre la migración peruana en el periodo comprendido entre los años 2006-2009 indican un retroceso en la llegada de peruanos al Perú, a excepción de los provenientes de Chile. De otro lado, DIGEMIN indica que cerca de 80427 personas han retornado al Perú entre los años 2007 a 2009; en su mayoría ha sido población femenina $(53,6 \%)$. Si observamos esta cifra por cada año se ve un

22 Mucha de la información presentada aquí ha formado parte del desarrollo de las consultorías privadas realizadas dentro del marco del Proyecto Migrandina. 
incremento paulatino de retornados: 21 747, 27547 y 31 133. En cuanto a la distribución etaria de los retornados hay una mayor frecuencia de los que se encuentran entre los 30 a 34 años de edad. Sobre el lugar de retorno se indica que es más frecuente la llegada de Chile y Estados Unidos:

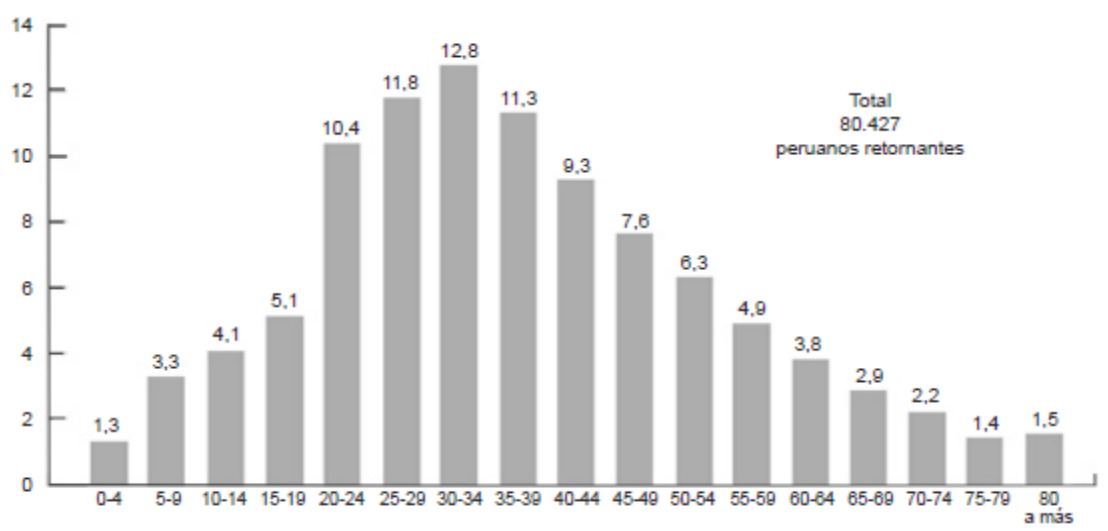

Gráfico 1. Perú, distribución porcentual de los nacionales retornados por grupo etario, 2007-2009

Fuente: Instituto Nacional de Estadística e Informática (2009: 43).

El estudio peruano sobre el retorno entre 2007 y 2009 también reporta datos acerca de la profesión de los llegados y dice:

Del total de profesionales que retornaron al país, la mayoría son profesores con $16,6 \%$, ingenieros $(13,9 \%)$, administradores de empresas $(10,5 \%)$, enfermeros $(6,3 \%)$, contadores $(6,0 \%)$, religiosos (4,5\%), abogados (4,4\%), médicos (4,1\%) economistas $(3,6 \%)$, entre las principales profesiones que declararon los retornantes al momento de ingresar al país. (Convenio de Cooperación INEI-DIGEMIN-IO, 2009: 48). 
España a través de sus organismos institucionales indica que en el caso de retornados participantes en programas de retorno implementados del Gobierno de España, en los años 2009 y 2011, han retornado más de 800 personas (163 a través del Programa de atención social; 652 con el programa APRE y 24 con el programa de retorno productivo).

En líneas arriba se había mencionado que el MTPE tiene dos sistemas para registrar a peruanos con voluntad de migrar.aA la fecha de la investigación se encontraron menos de 200 personas registradas.. Sobre esta pequeña base de datos del MTPE se puede informar que en su mayoría son las mujeres que han usado la plataforma de retorno, entre 35 a 55 años de edad. Las mismas que han residido en España, EEUU, Japón, Colombia entre otros. Otras características resaltantes es que poseen doble nacionalidad; cuentan con estudios laborales; en cuanto a sus expectativas laborales en el Perú piensan emprender un negocio; y otras trabajar como dependientes.

Según el estudio de la IOM²3-DIGEMIN (1990-2009) se indica que hay más de 80000 casos de peruanos retornados siendo las siguientes las principales características: $53.6 \%$ mujeres; estando en mayor porcentual entre el cohorte de 30 a 34 años de edad; en su mayoría son solteras (71.5\%); en cuanto a los países de procedencia destacan Chile, EEUU y España; un $12 \%$ son profesionales (docentes, ingenieros; administradores de empresas; enfermeros; contadores; abogados; médicos y economistas)

De otro lado, el gobierno de España ha impulsado programas de retorno voluntario para retornar a los países de origen. Entre los datos que se destacan se puede indicar al «Programa de retorno de atención social»,

23 Metodológicamente, cabe decir que este estudio aborda el cálculo del número de personas peruanas registradas por los diferentes puntos de control migratorio que han salido del país y que no registraron su retorno, pasados seis meses de su salida 
el mismo que busca facilitar a las personas extranjeras, especialmente vulnerables, la posibilidad de retornar voluntariamente desde España a sus países de procedencia, para su reintegración. Desde 2003 han participado en este programa 245 personas de nacionalidad peruana. En cuanto al «Programa de retorno productivo» tiene por objetivo favorecer el regreso al país de origen con enfoque productivo, capacitando a las personas interesadas para la creación de proyectos empresariales de pequeñas empresas familiares antes de su salida de España. Entre el año 2010 y 2011 han participado veinticuatro personas de nacionalidad peruana en este tipo de programa de retorno, destacando un claro predominio de los varones sobre las mujeres (un $85 \%$ frente a un 15\%). Cuya edad promedio es de 44 años de edad; el $58 \%$ posee estudios secundarios, un $33 \%$ estudios universitarios medios y ninguno de los participantes posee estudios universitarios superiores. Con respecto al «Programa de retorno de ayudas complementarias al Abono Acumulado y Anticipado de la Prestación de Desempleo» es un programa implementado en relación con la actual situación de crisis económica. El número de solicitudes de personas de nacionalidad peruana tramitadas hasta el primer semestre de 2011 fue de 1 744, siendo según estos datos, la cuarta nacionalidad cuantitativamente más relevante, tras Ecuador, Colombia y Argentina. De otro lado, existe el Programa de Voluntario de Retorno para personas desempleadas con derecho a prestación por desempleo, que gestiona un programa de ayudas complementarias para el viaje de personas que participan en este programa de retorno voluntario, en el que han participado, desde 2009, 652 personas de nacionalidad peruana. El perfil tipo de participantes de esta nacionalidad en este programa es el de un varón mayor de treinta y cinco años de edad que regresa con familiares a cargo. 
Los datos concluyen que no hay un solo prototipo de peruano a retornar, sino una variedad de peruanos que han desarrollado algún tipo de cualificación en los lugares de acogida, que muchas veces la decisión de retorno va más allá del tema laboral.

\section{POLÍTICAS PÚBLICAS EN CUANTO AL RETORNO: EL BALANCE}

- El Estado Peruano cuenta con programas y acciones directas para el proceso del ciclo migratorio, en especial, se han diseñado mecanismos para el retorno. Sin embargo se observa que estos no cuentan con una coordinación efectiva, sobre todo, a manera interinstitucional, lo que no facilita la implementación y el seguimiento de iniciativas. - En cuanto al retorno también se puede indicar que el concepto que se maneja es un "retorno generalizad"», pues cada una de las iniciativas tiene un sentido global y las mismas no son coordinadas entre Ministerios, encontrando en algunos casos duplicidad. Muchos Ministerios sobre la base da su experiencia, competencias y funciones diseñan y ejecutan instrumentos con respecto al retorno los mismos que no son coordinados. Esta situación, ocasion distorsión de información para el peruano que desea retornar.

- La Superintendencia Nacional de Migraciones es la encargada a través de la Tarjeta Andina de Migración (TAM) de producir información estadística; la misma que no coordina funciones ni con los servicios consulares ni con el MTPE ni el MR,. Este hace imposible una buena gestión de la información en cuanto al retorno; por ejemplo la Plataforma de Retorno no considera los beneficios de la nueva Ley de Incentivos Migratorios y también en la TAM se carece de información para realizar perfiles del retornado. 
- En general, el gobierno peruano ha realizado esfuerzos en materia del retorno que son importantes, pero no suficientes para lograr una política integral, articulada y sostenible.

-El Ministerio de Trabajo y Promoción del Empleo ha diseñado instrumentos ligados exclusivamente a la reintegración laboral del retornado. Es importante más bien trabajar en el diseño de perfiles para poder incluir a los potenciales retornados acorde a su cualificación y experiencia en los sectores especializados; el CONCYTEC es otra institución que está trabajando proyectos en materia de retorno, sobre todo, de científicos altamente cualificados. Sin embargoo igual que en el caso anterior se encuentra todavía un divorcio entre las instituciones para trabajar con fines conjuntos. Aún falta incentivar un mayor uso de información para el diseño de políticas prácticas. Asimismo; se necesita realizar actividades de seguimiento a fin de coordinar tareas en el propio ministerio, dado que existen dos servicios de atención al retornado (Plataforma de retorno y la orientación al migrante).

-Es importante que, sobre todo, los servicios del Ministerio se puedan conocer mucho más entre las asociaciones $\mathrm{d}$, los peruanos $\mathrm{y}$ organizaciones que trabajan con migrantes. Asimismo, es necesarie incorporar los servicios en las páginas web de los consulados.

-Es importante que el Estado diseño procesos de atención al retornado desde el punto de vista de un enfoque multidimensional, considerando que es un retorno integral de la persona. El Estado debe pensar el desarrollar mecanismos que incluyan un trabajo en red, con enfoque participativo y de género, donde el seguimiento y 
PROCESO MIGRATORIO DE LOS PERUANOS:

la evaluación constante del programa de retorno ayuden a realizar retroalimentaciones que conducen a la calidad del proceso. 


\section{BIBLIOGRAFÍA}

Alfaro, Yolanda y Lorena Yzaguirre 2010

«Migración y perspectivas de retorno. Estado de la Situación». Documento de trabajo. S.e.

Ballesteros, $\mathrm{X}$.

2006

Migración de retorno en espacios globales. Aproximaciones teóricas. Migración, trabajo y medio ambiente, acercamientos teóricos en las ciencias sociales desde el Caribe mexicano. Sierra, Ligia, Robertos, Julio (eds.) México: Universidad de Quintana Roo; Plaza y Valdés.

Berninghaus, S. y H.G Seifert-Vogt

1989

«Temporary vs permanent migration: a decision theoretical approach». Journal of polulation Economisc, Año 3, Vol. 1, pp.195-211.

Berger, J, y J. Mohr

1975

A Seventh Man. New York: Viking Press.

Bertossi, Christoph

2010

The State strives to shape "chosen" immigration.

Documento de Trabajo, Roma: CESPI, 2008, 3-4. Documento disponible en Centro Studi di Politica Internazionale <http://www.cespi. 
PROCESO MIGRATORIO DE LOS PERUANOS:

it/WPMIG/Contry\%20mig-FRANCIA.pdf>.

Consulta hecha en 20/10/2012.

Castles, Stephen, y G. Kosack

1973

Inmigrant workers and Class Structure in Western Europe. Londres: Oxford University Press.

Cesare, F.P.

1974

«Expectations and reality. A case study of return migration from the United States to Southern Italy». International Migration Review 8, N. 2: Policy and research on migration. Canadian and World Perspectives, pp. 245-262.

Congreso de la República 2004

LeyN.28182de incentivos migratorios. Documento disponible en <http://www2.congreso.gob.pe/ Sicr/TraDocEstProc/TraDoc_expdig_2006.nsf/ Dv07>.

2005

Reglamento de la Ley N. 28182 - Ley de Incentivos Migratorios. Que fue aprobado a través del DECRETO SUPREMO N. 028-2005EF. Documento disponible en <http://www2. congreso.gob.pe/Sicr/TraDocEstProc/TraDoc_ expdig_2006.nsf/Dv07>.

2010

Ley N. 29508 que restablece la vigencia de la Ley N. 28182 de incentivos migratorios. Documento disponible en <http://www2.congreso.gob.pe/ 
Sicr/TraDocEstProc/TraDoc_expdig_2006.nsf/ Dv07>.

DuRAND, Jorge

2004

2005

«Ensayo Teórico sobre la migración de retorno». En Cuadernos Geográficos, N. 35, pp. 103-116.

2005 «Ensayo teórico sobre la migración de retorno: el principio del rendimiento decreciente». Contribuciones al análisis de la migración internacional y el desarrollo regional en México. En Delgado Wise, Raúl y Beatrice Knerr (coordinadores), N. 35, pp. 103-116.

«De traidores a héroes. Políticas emigratorias en un contexto de asimetría de poder». En Delgado Wise, Raúl y Beatrice KNeER, (coordinadores) Contribuciones al análisis de la migración internacional y el desarrollo regional en México. Mexico: Miguel Ángel Porrúa/Universidad Autónoma de Zacatecas. pp.15-38.

Ferrero, Ruth, y Ana López

"Nuevas dinámicas de gestión de las migraciones en Espańa: el caso de los acuerdos bilaterales de trabajadores con países de origen.» Revista del Ministerio de Trabajo e Inmigración de España (MTIN), N. 80, p. 120. 
PROCESO MIGRATORIO DE LOS PERUANOS:

Franco Aguilar, José 2010

«Los que regresan: migración de retorno en la región de los Altos de Jalisco». Seminario: Movilidad espacial de la población y migración. Presentado en la Maestría en Población y Desarrollo de la Facultad Latinoamericana de Ciencias Sociales. México. Agosto.

GaIllard, A.M.

1994

Migration return: a bibliographical overview. Nueva York: Center for Migration Studies.

Glaser, William A. y G. Christopher Habers

1974

"The Migration and Return of Professionals». International Migration Review, Vol. 8, N. 2, pp. 227-224.

GMELCH, George

1980

«Return Migration». Annual Review of Anthropology, Vol. 9, pp. 135- 159.

Goldstein, Sidney 1964

«The Extent of Repeated Migration: An Analysis Based on the Danish Population Register». Journal of the American Statistical Association, Vol. 59, N. 308, pp. 1121-1132. 
HiguCHI, N.

2009

Mendiola, Mariana 2009

Miller, Mark J.

1995

OCAÑa, Juan Carlos 2000
Mass Unemployment of Japanese Latin american as a Disaster Made by Humans: The Consequences of Labour-Market Flexibilisation During the Economic Crisis in Japan. Oxford: Centre on Migration Policy and Society (COMPAS).

«El retorno de los emigrantes peruanos. Un análisis de los mecanismos de gestión del retorno en el Perú desde el enfoque de los derechos humanos». Revista del Observatorio Andino de Migraciones TukuyMigra, Pontificia Universidad Católica del Perú, N. 1, pp. 2-15.

Employer Sanctions in France: From the Campaign Against Ilegal Alien Employment to the Campaign Against Ilegal Work. Delaware: Universidad de Delaware, Comission on Immigration Reform.

«La Unión Europea: el proceso de integración y la ciudadanía europea». Documento disponible en <http://clio.rediris.es/udidactica/amsterdam. htm>. Consulta hecha en 01/02/2012. 
PROCESO MIGRATORIO DE LOS PERUANOS:

Plewa, Piotr

2009

"Voluntary Return Programmes: Could they assuage the effects of the economic crisis?» Documento de Trabajo, Centre on Migration, Policy and Society (COMPAS). Oxford: Universidad de Oxford, p. 29.

ReICHERT, Joshua, y Douglas Massey

1982

"Guestworker programs: Evidence from Europe and the United States and some implications for U.S. polic.». Population Research and Policy Review, Vol. 1, N. 1, p. 3.

Rist, Ray

1979

"Guestworkers and post-world war II European migration.». Studies in Comparative International Development (SID) 14, N. 2, pp. 29-30.

ROGERS, R.

1998

"Migrations return policies and countries of origin». En Hailbronner Kay, David A. Martin y Hiroshi Мотомura. Immigration Admission, Vol. 3. United States: Berghahn Books, pp. 446204.

Organización Internacional para las Migraciones, el Instituto NACiONAL de Estadística E INFORMÁtica 2009

«Perú: Migración Internacional en las Familias Peruanas y Perfil del Peruano Retornante». 
Documento disponible en <http://www.mintra. gob.pe/migrante/pdf/migracion_internacional_ failias_peruanas.pdf $>$. Consulta hecha en 24/01/2014.

The Organisation for Economic Co-operation and Development 2010

International Migration Outlook (SOPEMI). Paris: OECD Publishing. 